\title{
Initial state with shear in peripheral heavy ion collisions
}

\author{
V. K. Magas, ${ }^{1,2}$ J. Gordillo, ${ }^{1}$ D. Strottman,,${ }^{3,4}$ Y. L. Xie,,${ }^{3,5, *}$ and L. P. Csernai ${ }^{3}$ \\ ${ }^{1}$ Departament de Fisica Quantica i Astrofisica, Universitat de Barcelona, 08028 Barcelona, Spain \\ ${ }^{2}$ Institut de Ciencies del Cosmos, Universitat de Barcelona, 08028 Barcelona, Spain \\ ${ }^{3}$ Institute of Physics and Technology, University of Bergen, Allegaten 55, 5007 Bergen, Norway \\ ${ }^{4}$ Los Alamos National Laboratory, Los Alamos, 87545 New Mexico, USA \\ ${ }^{5}$ Frankfurt Institute for Advanced Studies, Ruth-Moufang-Str. 1, 60438 Frankfurt am Main, Germany
}

(Received 1 December 2017; published 8 June 2018; corrected 19 July 2018)

\begin{abstract}
In the present work we propose a new way of constructing the initial state for further hydrodynamic simulation of relativistic heavy ion collisions based on Bjorken-like solution applied streak by streak in the transverse plane. Previous fluid dynamical calculations in Cartesian coordinates with an initial state based on a streak by streak Yang-Mills field led for peripheral higher energy collisions to large angular momentum, initial shear flow and significant local vorticity. Recent experiments verified the existence of this vorticity via the resulting polarization of emitted $\Lambda$ and $\bar{\Lambda}$ particles. At the same time parton cascade models indicated the existence of more compact initial state configurations, which we are going to simulate in our approach. The proposed model satisfies all the conservation laws, including conservation of a strong initial angular momentum, which is present in noncentral collisions. As a consequence of this large initial angular momentum we observe the rotation of the whole system as well as the fluid shear in the initial state, which leads to large flow vorticity. Another advantage of the proposed model is that the initial state can be given in both $[\mathrm{t}, \mathrm{x}, \mathrm{y}, \mathrm{z}]$ and $[\tau, x, y, \eta]$ coordinates and thus can be tested by all $3+1 \mathrm{D}$ hydrodynamical codes which exist in the field.
\end{abstract}

DOI: 10.1103/PhysRevC.97.064903

\section{INTRODUCTION}

About 15 years ago a nucleus-nucleus initial state (IS) model was constructed [1,2] based on the longitudinal effective string rope model for realistic, $3+1 \mathrm{D}$ relativistic fluid dynamical models. This model preceded the early development of Quark-Gluon Plasma (QGP) research, but still it reflected correctly not only the energy-momentum, but also angular momentum conservation, initial shear flow, and local vorticity. Actually as a consequence of the large initial angular momentum present in the noncentral ultrarelativistic heavy ion collisions, the effective rotation of the whole fireball has been observed once the effective string rope model was applied to simulate $\mathrm{Pb}+\mathrm{Pb}$ collisions at ALICE@LHC [3]. Obviously such a rotation leads to large flow vorticity [4].

Several other initial state models neglected these basic features, but recent experimental and theoretical developments indicate that angular momentum, local vorticity, and the subsequent particle polarization is observable and provides valuable information about the QGP. Recently, significant $\Lambda$ polarization was detected and analyzed in detail in the RHIC BES program $[5,6]$. These results indicate that shear and vorticity should not be neglected if we wish to account for the observed polarization.

Several parton kinetic and field theoretical models were recently implemented to describe the IS, providing a rather different initial state configuration, especially for noncentral collisions [7-12]. While in peripheral collisions in Refs. [1,2],

*Corresponding author: yi.long.xie.china@gmail.com the off-center streaks were assumed to have relatively weak fields and therefore showed large longitudinal extent, the present kinetic models show a more compact IS, where the streaks away from the center are more compact and experience stronger fields. We can see this in Fig. 1.

This figure is a result of the simulation of $\mathrm{Au}+\mathrm{Au}$ collisions at the energy $\sqrt{s_{\mathrm{NN}}}=200 \mathrm{GeV}$ of $40-50 \%$ centrality by means of a multiphase transport (AMPT) model [7]. In AMPT the initial partons are generated by the HIJING and ZPC models [7]. A string-like mechanism of initial parton production is used in the model: in AMPT the partons are clustered according to their transverse coordinates into string-like objects. The partons of the same cluster are plotted in the same color and size. The string position and length fluctuations are demonstrated more clearly by replacing the partons in the same cluster with line segments (strings). The varying colors indicate the varying transverse clusters. The density of strings indicates the local energy density; see Fig. 1.

As we can see Fig. 1 shows an example of an initial state configuration, which is more compact than the afore mentioned early initial state models $[1,2]$. Note that the offcenter side streaks are actually shorter than the central streaks, and at the same time they have obviously moved forward and backward indicating angular momentum conservation and significant local shear. The thin magenta external contour line, inserted in Fig. 1, surrounding the matter distribution, shows the characteristic shape of the initial state obtained in the AMPT model, which provides us a guidance to form a more compact initial state model with fixed longitudinal extent of the projectile/target side peripheral streaks. 


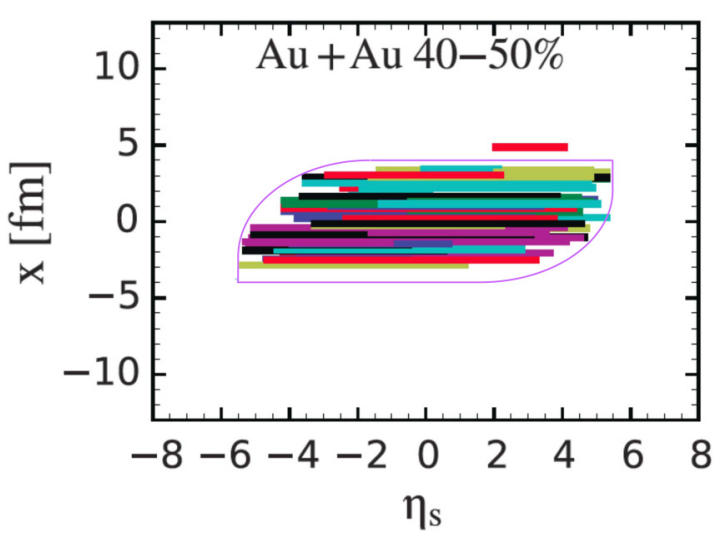

FIG. 1. Initial state strings indicate the spatial distribution with fluctuating initial conditions from a multiphase transport (AMPT) model [7]. The model simulates $\mathrm{Au}+\mathrm{Au}$ collisions at the energy of $\sqrt{s_{\mathrm{NN}}}=200 \mathrm{GeV}$ of centrality percentage $40-50 \%$ in the reaction plane in $\left[x ; \eta_{s}\right]$ coordinates. The spatial energy distribution remains compact and the off-center side streaks are actually shorter than the central streaks. At the same time the off-center side streaks have obviously moved forward or backward indicating angular momentum conservation and significant local shear. The inserted thin magenta line surrounding the matter distribution shows the characteristic shape of the initial state obtained in the AMPT model.

Those models that account for the initial shear and vorticity $[4,8,13-15]$ could predict and study the observed polarization.

These developments make it timely that in view of new experimental and theoretical developments we need to revisit the early IS model, with the aim of keeping all basic features as local shear, angular momentum conservation, and local vorticity, while adapting to the developments in parton kinetic [9-11] and field dominance [12] models. Furthermore, as several field theoretical models have been developed recently in the proper-time and space-time rapidity, Milne coordinates $[\tau, x, y, \eta]$, we also present the model in the same way to make it useful for other approaches. However, we will continue to use fluid dynamical models in Cartesian coordinates, $[t, x, y, z]$, as, e.g., the particles in cell relativistic model (PICR), since in these codes the numerical effects are well studied.

\section{HEAVY ION COLLISIONS AS A SET OF INDEPENDENT SLAB-SLAB COLLISIONS}

Let us consider a peripheral heavy ion collision at highly relativistic energies. The projectile and target are strongly Lorentz contracted before the collision, while the parton momentum distributions of the projectile and target are strongly Lorentz elongated.

We divide up the transverse plane into cells of less than $1 \mathrm{fm}^{2}$ size. The corresponding elements of the projectile and target hit and interpenetrate each other. The transverse expansion can be ignored for the first moments of the collision, and thus, at the beginning, one can describe the nucleus-nucleus collision as a set of independent, initial slab-slab collisions, corresponding to the same transverse coordinates. One or two $\mathrm{fm} / \mathrm{c}$ after the first contact the partons from the short, Lorentz contracted, pre collision projectile, and target slabs will form a streak, which is about $2-4 \mathrm{fm}$ long.

Throughout this paper we shall assume that the projectile will have an initial positive (forward) momentum and the target will have a negative momentum in the center-of-mass (c.m.) system.

Due to the large momentum spread of the initial partons the resulting streaks will have a mixture of projectile and target partons at each point of the streak. In peripheral collisions in a given slab-slab collision there will be a projectile/target asymmetry, except at the central streaks corresponding to $x=$ $x_{c}$. The final streaks will have a finite longitudinal momentum, which can be calculated using the longitudinal momentum conservation from momenta of the two original slabs. Streaks on the projectile side of the transverse plane $\left(x>x_{c}\right)$ will have a forward momentum, while on the target side $\left(x<x_{c}\right)$ a backward one. Thus, each streak will have its own c.m. reference system. Neighboring streaks may have an initial shear.

This compact system will have initially $(\sim 1-2 \mathrm{fm} / \mathrm{c})$ a nonzero angular momentum. Its partons will be mixed from the projectile and target. The chromomagnetic forces (string tension) will attract the leading partons. So, the system will not expand with the speed of light but will be held back by the fields. The original Lorentz elongation of the momentum distribution and the field attraction will lead to an initial parton distribution, which will be close to uniform, as both the target and projectile partons can populate the whole length of the moving streak [12], as assumed in the Bjorken model.

Let us consider projectile and target slabs colliding head on with each other at a given transverse point $\left[x_{i}, y_{i}\right]$. The main ansatz of this work is to assume that the Bjorken model can be applied to describe these slab-slab collisions during the initial stage of the reaction. This means that the resulting streak of matter has a longitudinal rapidity profile as in the Bjorken flow expansion, contrary to the rapidity profiles used in Refs. $[1,2,16,17]$. However, each of these streaks will be described by the Bjorken flow in its own c.m. frame. The overall reaction volume, i.e., all the streaks together, can be described in the Lab frame, where for each such streak $i$ we can construct, as we shall see later, a starting point $\left[t_{i 0}, z_{i 0}\right]$.

From the initial geometry we know for each final streak $i$, at a transverse point $\left[x_{i}, y_{i}\right]$, the total baryon charge, the total kinetic energy, and the total momentum in the longitudinal direction. For simplicity we will drop the subindex $i$ in the rest of this section, since here we will only be interested in one transverse position. Later, to describe the whole collision, all the quantities introduced and derived in this section will have the subindex of the corresponding transverse position.

Thus, we want to describe collisions of two slabs of the nuclear matter with $N_{1}, E_{1}, P_{1 z}$ and $N_{2}, E_{2}, P_{2 z}$ for projectile and target, respectively. The precollision projectile slab moves with the beam rapidity $y_{0}$ while the target slab with $-y_{0}$. In Fig. 2 we show the slab-slab collision (asymmetric in the general case). The first contact happens at $\left(t_{0}, z_{0}\right)$, and at the proper time $\tau_{0}$ the resulting streak stretches from $t_{\min }, z_{\min }$ to $t_{\max }, z_{\max }$.

The transformation between the usual Cartesian coordinates $x^{\mu}=(t, x, y, z)$ and Milne coordinates $\tilde{x}^{\mu}=(\tau, x, y, \eta)$ is 


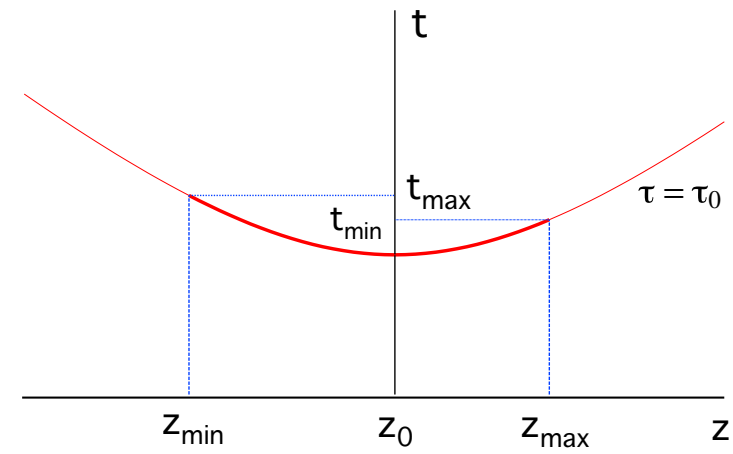

FIG. 2. Space-time sketch of a slab-slab collision in the collider laboratory frame. At proper time $\tau_{0}$ after the collision, the resulting streak stretches from $t_{\min }, z_{\min }$ to $t_{\max }, z_{\max }$.

given as

$$
\begin{aligned}
t-t_{0} & =\tau \cosh \eta, \\
z-z_{0} & =\tau \sinh \eta, \\
\tau & =\sqrt{\left(t-t_{0}\right)^{2}-\left(z-z_{0}\right)^{2}}, \\
\eta & =\frac{1}{2} \ln \left(\frac{t-t_{0}+z-z_{0}}{t-t_{0}-\left(z-z_{0}\right)}\right)=\operatorname{Artanh} \frac{z-z_{0}}{t-t_{0}},
\end{aligned}
$$

where $\eta$ is the space-time rapidity. Consequently,

$$
\begin{aligned}
d t & =\cosh \eta d \tau+\tau \sinh \eta d \eta, \\
d z & =\sinh \eta d \tau+\tau \cosh \eta d \eta, \\
d z d t & =\tau d \eta d \tau .
\end{aligned}
$$

In case of the longitudinal Bjorken scaling flow, the local flow velocity of matter is

$$
u^{\mu}=x^{\mu} / \tau=(\cosh \eta, 0,0 \sinh \eta) .
$$

Thus, the velocity of the Bjorken flow at point $(t, z)$ is

$$
v_{z}=\frac{z-z_{0}}{t-t_{0}}
$$

and for the streak ends we can write

$$
\begin{aligned}
& \eta_{\max }=\operatorname{Artanh} v_{\max }=\operatorname{Artanh} \frac{z_{\max }-z_{0}}{t_{\max }-t_{0}}, \\
& \eta_{\min }=\operatorname{Artanh} v_{\min }=\operatorname{Artanh} \frac{z_{\min }-z_{0}}{t_{\min }-t_{0}},
\end{aligned}
$$

or in other words

$$
\begin{aligned}
& z_{\max }=z_{0}+\tau_{0} \sinh \eta_{\max }, \\
& z_{\min }=z_{0}+\tau_{0} \sinh \eta_{\min } .
\end{aligned}
$$

\section{A. Conservation Laws}

Following the philosophy of the Bjorken model we assume that each streak, at the moment when its proper time $\tau$ is equal to $\tau_{0}$, contributes to the initial state at local thermal equilibrium. Then it evolves further according to the hydrodynamic Bjorken equations. The main characteristic of this $\tau=\tau_{0}$ state is that it is constant as a function of $\eta$, while the local flow four-velocity is given by Eq. (5). The initial energy and baryon densities can be found from the conservation laws.

The $\tau=$ const. hypersurface normal four-vector is given as

$$
d^{3} \Sigma_{\mu}^{(t, z)}=\tau(\cosh \eta, 0,0,-\sinh \eta) d x d y d \eta=\tau A u_{\mu} d \eta,
$$

where $A$ is the transverse cross section of the streak (in the $[x, y]$ plane).

The net baryon four current for a streak is $N^{\mu}=n u^{\mu}$, and thus the net baryon number crossing a constant $\tau$-hypersurface element is

$$
d N=d^{3} \Sigma_{\mu} N^{\mu}=n \tau A d \eta .
$$

Thus, the baryon number conservation for a streak, assuming uniform $\eta$-distribution, gives

$$
N=N_{1}+N_{2}=\tau_{0} n\left(\tau_{0}\right) A\left[\eta_{\max }-\eta_{\min }\right],
$$

where $\left(N_{1}+N_{2}\right)$ is an invariant scalar given by the Projectile (1) and Target (2) baryon charge contribution to a given streak, and the difference, $\left(\Delta \eta=\eta_{\max }-\eta_{\min }\right)$, should also be a boost invariant quantity.

The energy-momentum tensor is $T^{\mu \nu}=e u^{\mu} u^{v}-p \Delta^{\mu \nu}+$ $\pi^{\mu \nu}$, where $\Delta^{\mu \nu}=g^{\mu \nu}-u^{\mu} u^{\nu}$ is the projection tensor and $\pi^{\mu \nu}$ is the shear-stress tensor, both orthogonal to the flow velocity. Energy crossing the $\tau=$ const. hypersurface element is

$$
\begin{aligned}
d E & =d^{3} \Sigma_{\mu} T^{0 \mu}=\tau A\left[e u^{0} u^{\mu}-p \Delta^{0 \mu}+\pi^{\mu 0}\right] u_{\mu} d \eta \\
& =\tau A e u^{0} d \eta=\tau A e \cosh \eta d \eta .
\end{aligned}
$$

Integrating this between $\eta_{\max }$ and $\eta_{\min }$, leads to

$$
E=E_{1}+E_{2}=\tau_{0} e\left(\tau_{0}\right) A\left(\sinh \eta_{\max }-\sinh \eta_{\min }\right) .
$$

Note that both $E_{1}+E_{2}$ and $\left(\sinh \eta_{\max }-\sinh \eta_{\min }\right)$ are frame dependent. Nevertheless, the equations for $N$ and $E$ have the same form in any boosted frame.

Similarly, for the longitudinal momentum component we have

$$
d P_{z}=d^{3} \Sigma_{\mu} T^{z \mu}
$$

and it follows that

$$
\begin{aligned}
P_{z} & =\tau_{0} A \int\left[e u^{z} u^{\mu}-p \Delta^{z \mu}+\pi^{\mu z}\right] u_{\mu} d \eta \\
& =\tau_{0} A \int e u^{z} d \eta=\tau_{0} A e \int \sinh \eta d \eta,
\end{aligned}
$$

and so

$$
P_{z}=P_{1 z}-P_{2 z}=\tau_{0} A e\left(\cosh \eta_{\max }-\cosh \eta_{\min }\right) .
$$

The above equations can be given in a more compact form if we introduce for each streak instead of $\eta_{\max }$ and $\eta_{\min }$ two other quantities, namely semidifference $\Delta \eta / 2$ and c.m. rapidity $\langle\eta\rangle$, given as

$$
\begin{aligned}
\frac{1}{2} \Delta \eta & =\frac{\eta_{\max }-\eta_{\min }}{2}, \\
\langle\eta\rangle & =\frac{\eta_{\max }+\eta_{\min }}{2} .
\end{aligned}
$$


With these parameters, from Eqs. (11), (13), and (16), it follows that

$$
\begin{aligned}
N & =\tau_{0} n\left(\tau_{0}\right) A \Delta \eta, \\
E & =2 \tau_{0} e\left(\tau_{0}\right) A \sinh (\Delta \eta / 2) \cosh (\langle\eta\rangle), \\
P_{z} & =2 \tau_{0} e\left(\tau_{0}\right) A \sinh (\Delta \eta / 2) \sinh (\langle\eta\rangle) .
\end{aligned}
$$

Comparing Eqs. (20) and (21) we find an expression for c.m. rapidity:

$$
\langle\eta\rangle=\operatorname{Artanh} \frac{P_{z}}{E}
$$

\section{THE IMPLEMENTATION OF THE MODEL}

One must select an initial proper time parameter, $\tau_{0}$, for the model which can be chosen relatively freely. In the literature the typical values of the Bjorken initial proper time $\tau_{0}$ vary from $0.1 \mathrm{fm}$ to a few $\mathrm{fm}$. According to our assumptions the fluid elements show a Bjorken-type scaling expansion where the flow rapidity equals the rapidity coordinate, $\eta$, of a given fluid element of the streak in the rest frame of the streak (RFS). The streaks corresponding to different transverse coordinates, $\left[x_{i}, y_{i}\right]$, have in general different reference rest frames, $\operatorname{RFS}_{i}$, and different initial points $t_{i 0}$ and $z_{i 0}$.

\section{A. Central streak}

Let us now consider the central streak, denoted by index $i=c$. For this streak the collider c.m. frame and the rest frame of the streak (RFS) are the same, and correspondingly $\tau_{0}=t_{0}$. The total energy of this streak is then $E_{c}$, and its momentum is $P_{c z}=0$, and correspondingly, see Eq. (22), $\left\langle\eta_{c}\right\rangle=0$. Thus, for the central streak we have $-\Delta \eta_{c} / 2<\eta_{c}<\Delta \eta_{c} / 2$.

We choose also a coordinate system so that the first contact of the target and projectile slabs for the central streak happens at $t=0$ and $z=0$. Thus, for the central streak the starting coordinates for the Bjorken solution are $t_{0, c}=0$ and $z_{0, c}=0$, while these have to be calculated for the peripheral streaks.

Then at $\tau_{c}=\tau_{0}$ the length of the central streak is $\Delta z_{c}=$ $z_{c-\max }-z_{c-\min }=2 \tau_{0} \sinh \left(\Delta \eta_{c} / 2\right)$. The extension of the central streak in the geometrical rapidity space, $\Delta \eta_{c}$, is one of our free parameters.

The energy density of the central streak at $\tau_{c}=\tau_{0}$ is given by Eq. (13):

$$
e_{c}\left(\tau_{0}\right)=E_{c} /\left[2 \tau_{0} A \sinh \left(\Delta \eta_{c} / 2\right)\right] .
$$

Also, once $\tau_{0}$ and $\Delta \eta_{c} / 2$ are set, using Eq. (1) we get the position of the forward edge of the central streak at

$$
\begin{aligned}
& z_{\max }^{c}=\tau_{0} \sinh \left(\Delta \eta_{c} / 2\right), \\
& t_{\max }^{c}=\tau_{0} \cosh \left(\Delta \eta_{c} / 2\right) .
\end{aligned}
$$

The position of the back edge can be calculated the same way, $t_{\min }^{c}=t_{\max }^{c}$ and $z_{\min }^{c}=-z_{\max }^{c}$.

\section{B. Peripheral streaks}

At finite impact parameter the asymmetry of the projectile and target side leads to a finite momentum, $P_{i z}$, for the peripheral streak $i$. Unlike in the usual approach, we do not set the origin of all streak hyperbolae to the same point (as the central streak); instead we make two assumptions:

(a) that in the collider c.m. frame the leading edge of the projectile $(P)$-side streaks will be aligned uniformly at the moment $\tau_{i}=\tau_{0}$, i.e., the $z_{\max }^{i, P}$ values will be the same on the projectile side, for all $i, P$-s,

$$
z_{\max }^{i, P}=z_{\max }^{c}
$$

(and on the target $(T)$-side, for all $i, T$-s the $z_{\min }^{i, T}=z_{\min }^{c}$ ). This reflects the behavior of the parton kinetic models as shown in Fig. 1;

(b) that at the moment $\tau_{i}=\tau_{0}$ in the corresponding $\operatorname{RFS}_{i}$,

$$
e_{i}\left(\tau_{0}\right)=e_{c}\left(\tau_{0}\right)=\text { const. }
$$

for all streaks $i$. This is in contrast to work in Refs. [1,2].

In the local rest frame of the $i$ th peripheral streak, $\mathrm{RFS}_{i}$, the streak momentum vanishes, while in the collider c.m. frame it is $P_{i z}$ given by the precollision parameters, and thus the streak rapidity in the collider c.m. frame is given by Eq. (22),

$$
\left\langle\eta_{i}\right\rangle=\operatorname{Artanh} \frac{P_{i z}}{E_{i}} .
$$

Based on Eqs. (20) and (26)

$$
\frac{1}{2} \Delta \eta_{i}=\operatorname{Arsinh}\left[\frac{E_{i}}{2 \tau_{0} e_{c}\left(\tau_{0}\right) A \cosh \left(\left\langle\eta_{i}\right\rangle\right)}\right] .
$$

Knowing $\left\langle\eta_{i}\right\rangle$ and $\Delta \eta_{i}$ means that $\eta_{i}$ varies between $\eta_{\min }^{i}=$ $\left\langle\eta_{i}\right\rangle-\frac{\Delta \eta_{i}}{2}$ and $\eta_{\max }^{i}=\left\langle\eta_{i}\right\rangle+\frac{\Delta \eta_{i}}{2}$, and the end points of streak $i$ on the $P$ side will be

$$
\begin{aligned}
& z_{\max }^{i, P}=z_{\max }^{c}, \\
& t_{\max }^{i, P}=t_{i 0}+\tau_{0} \cosh \eta_{\max }^{i},
\end{aligned}
$$

and

$$
\begin{aligned}
& z_{\text {min }}^{i, P}=z_{i 0}+\tau_{0} \sinh \eta_{\text {min }}^{i}, \\
& t_{\text {min }}^{i, P}=t_{i 0}+\tau_{0} \cosh \eta_{\text {min }}^{i},
\end{aligned}
$$

where $z_{i 0}$ and $t_{i 0}$ are still unknown.

Similarly, we can perform the calculation for the $T$-side streaks:

$$
\begin{aligned}
& z_{\text {max }}^{i, T}=z_{i 0}+\tau_{0} \sinh \eta_{\text {max }}^{i}, \\
& t_{\text {max }}^{i, T}=t_{i 0}+\tau_{0} \cosh \eta_{\text {max }}^{i},
\end{aligned}
$$

and

$$
\begin{aligned}
z_{\text {min }}^{i, T} & =z_{\text {min }}^{c}, \\
t_{\text {min }}^{i, T} & =t_{i 0}+\tau_{0} \cosh \eta_{\text {min }}^{i} .
\end{aligned}
$$

Now the baryon density at $\tau_{i}=\tau_{0}$ can be found from Eq. (19):

$$
n_{i}\left(\tau_{0}\right)=\frac{N_{i}}{\tau_{0} A \Delta \eta_{i}} .
$$

The previous description of assumption (a) was applicable for streaks in the reaction plane, i.e., for $y_{i}=0$ coordinate. For each $y_{i} \neq 0$ layer of streaks we define a new central streak with $P_{i z}^{c-y}=0$ and $\left\langle\eta_{i}^{c-y}\right\rangle=0$. Then following the assumption (b), 
Eq. (26), we can find the $\eta$ extension of this streak, similarly to Eq. (28),

$$
\frac{1}{2} \Delta \eta_{i}^{c-y}=\operatorname{Arsinh}\left(\frac{E_{i}^{c-y}}{2 \tau_{0} e_{c}\left(\tau_{0}\right) A}\right) .
$$

Then for all the noncentral streaks corresponding to the same coordinate $y$ we can repeat the above mentioned steps.

\section{Matching the leading $z$ and the mid- $t$ of the streaks}

As one can see in Fig. 3 if the space-time midpoints of the central and side streaks match, the back end of the side streak (red dashed hyperbola, starting at $\eta_{\min }$ ) may be very far from the central streak in the space-time, and the back end of the side streak may even be out of the light cone of the central streak. Instead one can assume that the front end of the side streak is at the same point as that of the central streak, but the time coordinate of the midpoint of the (red dashed) side streak, $t_{i \text { mid }_{P}}$, falls on the hyperbola of the (blue solid) central streak, as shown in Fig. 3. This midpoint corresponds to the mid-geometrical rapidity, i.e., $\eta_{i}=\left\langle\eta_{i}\right\rangle$ defined in Eq. (22):

$$
\begin{aligned}
t_{i \operatorname{mid}_{P}} & =t_{i 0}+\tau_{0} \cosh \left\langle\eta_{i}\right\rangle, \\
z_{i \operatorname{mid}_{P}} & =z_{i 0}+\tau_{0} \sinh \left\langle\eta_{i}\right\rangle .
\end{aligned}
$$

We now have to include the condition that the point $\left[t_{i \text { mid }_{p}}, z_{i \operatorname{mid}_{p}}\right]$ falls on the hyperbola of the central streak:

$$
\tau_{0}^{2}=t_{i \operatorname{mid}_{P}}^{2}-z_{i \operatorname{mid}_{P}}^{2} .
$$

With Eq. (35) this leads to the first connection between $t_{i 0}$ and $z_{i 0}$ :

$$
\tau_{0}^{2}=\left(t_{i 0}+\tau_{0} \cosh \left\langle\eta_{i}\right\rangle\right)^{2}-\left(z_{i 0}+\tau_{0} \sinh \left\langle\eta_{i}\right\rangle\right)^{2} .
$$

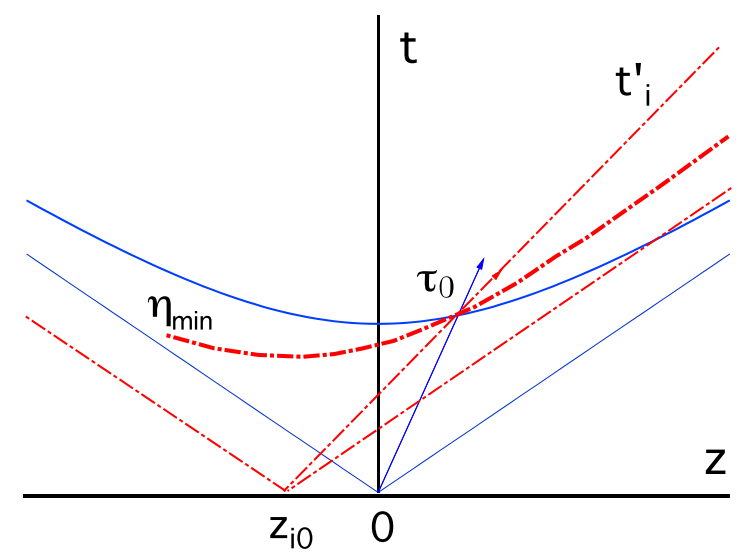

FIG. 3. Space-time sketch of the central streak (blue solid lines), and the $i$ th peripheral streak (red dashed lines) on the projectile side. The origins of the streaks are not identical, but at the proper time, $\tau_{0}$ and their leading edge position, $z_{\max }$, match. The two streaks cross each other in the space-time at the $t_{i}^{\prime}$ axis where both streaks have the same proper time $\tau_{0}$. This axis corresponds to the c.m. rapidity of the $i$ th peripheral streak, $\eta_{i}$. We can see that the local four velocity vectors are different for the two streaks causing shear and vorticity.
To find these two unknowns we have to add one more equation, which comes from our assumption (a):

$$
z_{\max }^{i, P}=z_{\max }^{c} .
$$

Thus, for the $P$ side we obtain

$$
z_{i 0}=z_{\max }^{c}-\tau_{0} \sinh \eta_{\max }^{i} .
$$

By keeping the longitudinal, forward $z$ positions of the streak ends at the same point, we know $z_{i 0}$ from Eq. (39). Then inserting $z_{i 0}$ into Eq. (37), we can get $t_{i 0}$. Thus, we get both $t_{i 0}$ and $z_{i 0}$ for each streak on the $P$ side.

The $T$ side can be calculated similarly by matching the back ends $\left(z_{\min }\right)$ of the side streaks.

Globally, for the whole collision, our assumptions lead to a rather compact IS in the space-time, as the centers of each side streak fall on the hyperbola of the central streak.

\section{Results for the first step of the model: Streaks at $\boldsymbol{\tau}_{i}=\boldsymbol{\tau}_{0}$}

To better illustrate how the model works we performed a calculation simulating a $\mathrm{Au}+\mathrm{Au}$ reaction at $100+100 \mathrm{GeV} / \mathrm{nucl}$ energy and impact parameter $b=0.5\left(R_{\mathrm{Au}}+R_{\mathrm{Au}}\right)=6.5 \mathrm{fm}$. The model parameters are fixed at $\tau_{0}=1.0 \mathrm{fm}$ and $\Delta \eta_{c}=2$, which leads to the energy density $e_{c}\left(\tau_{0}\right)=156.31 \mathrm{GeV} / \mathrm{fm}^{3}$. The results are shown in Figs. 4-10. Note that, strictly speaking, Figs. 4 and 5 serve only for a qualitative understanding

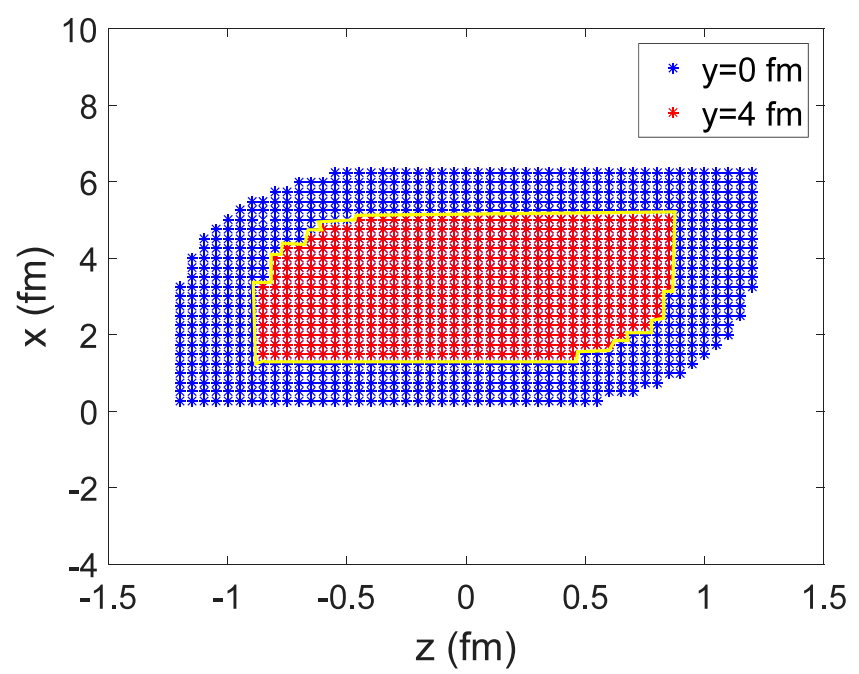

FIG. 4. The initial configuration of the streaks in the reaction plane, on the $\left[x, \eta, \tau_{i}=\tau_{0}=1 \mathrm{fm} / \mathrm{c}\right]$ hypersurface for $y=0$ (blue streaks with $z$ ranges of $[-1.2,1.2]$ ) and for $y=4 \mathrm{fm}$ (red streaks within the yellow closed line) overlaid. An assumption of our model is that the streak energy density is uniform and is the same for all streaks. The configuration is qualitatively similar to the parton cascade result shown in Fig. 1. This example is calculated for a $\mathrm{Au}+\mathrm{Au}$ reaction at $100+100 \mathrm{GeV} /$ nucl energy and impact parameter $b=0.5\left(R_{P b}+\right.$ $\left.R_{P b}\right)=6.5 \mathrm{fm}$, correspondingly, the $y=0$ plane crosses the $x$ axis at $x_{c}=3.25 \mathrm{fm}$. We have fixed our model parameters as $\tau_{0}=1.0 \mathrm{fm} / \mathrm{c}$ and $\Delta \eta_{c}=2$, which leads to the energy density $e_{i}\left(\tau_{0}\right)=e_{c}\left(\tau_{0}\right)=$ $156.31 \mathrm{GeV} / \mathrm{fm}^{3}$. Subsequent figures were calculated with the same reaction parameters. Note that this figure serves only for a qualitative understanding of the model, since each streak is plotted at the moment when its $\tau_{i}=\tau_{0}$. 


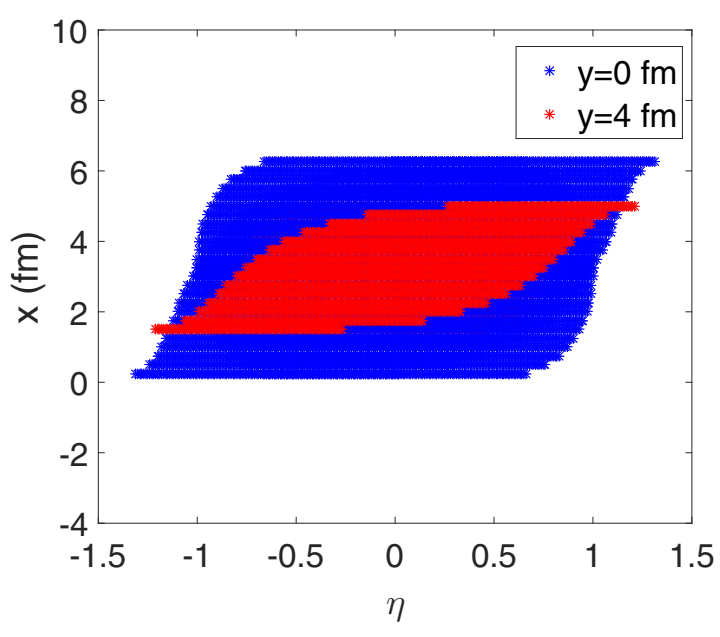

FIG. 5. The initial configuration of the streaks in the reaction plane, on the $\left[x, \eta, \tau_{i}=\tau_{0}=1 \mathrm{fm} / \mathrm{c}\right]$ hypersurface, plotted versus the geometrical rapidity $\eta$. Shown are the layers at $y=0$ (blue wider streaks) and at $y=4 \mathrm{fm}$ (red narrower streaks) overlaid. The streak energy density is uniform in $\eta$. The obtained configuration is also qualitatively similar to the parton cascade result shown in Fig. 1, but the top and bottom edges show a special behavior. This example is calculated for the same parameters as Fig. 4. Note that this figure serves only for a qualitative understanding of the model, since each streak is plotted at the moment when its $\tau_{i}=\tau_{0}$.

of the model, since all the streaks are plotted at the moment when their $\tau_{i}=\tau_{0}$. The method of constructing a proper model of the collision and presenting the different distributions at a given time in the Laboratory system or at one global proper time will be discussed in the next two sections.

Each of our final streaks has a scaling expansion flow in its own frame. Each streak is homogeneous and finite; all conservation laws, including the angular momentum, are

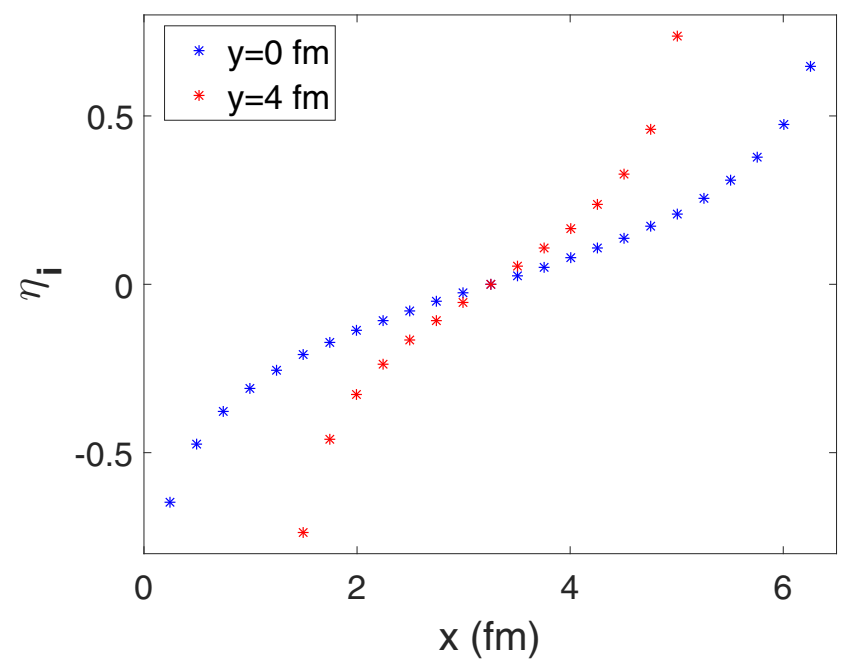

FIG. 6. The midrapidity of the streaks $\left\langle\eta_{i}\right\rangle$ in the reaction plane $[x, z]$ for different values of $x$, for $y=0$ (blue stars distributed among wider $x$ range $[0,6.5])$ and for $y=4 \mathrm{fm}$ (red stars distributed among narrow $x$ range $[1.5,5])$, for the same reaction and parameters listed in Fig. 4.

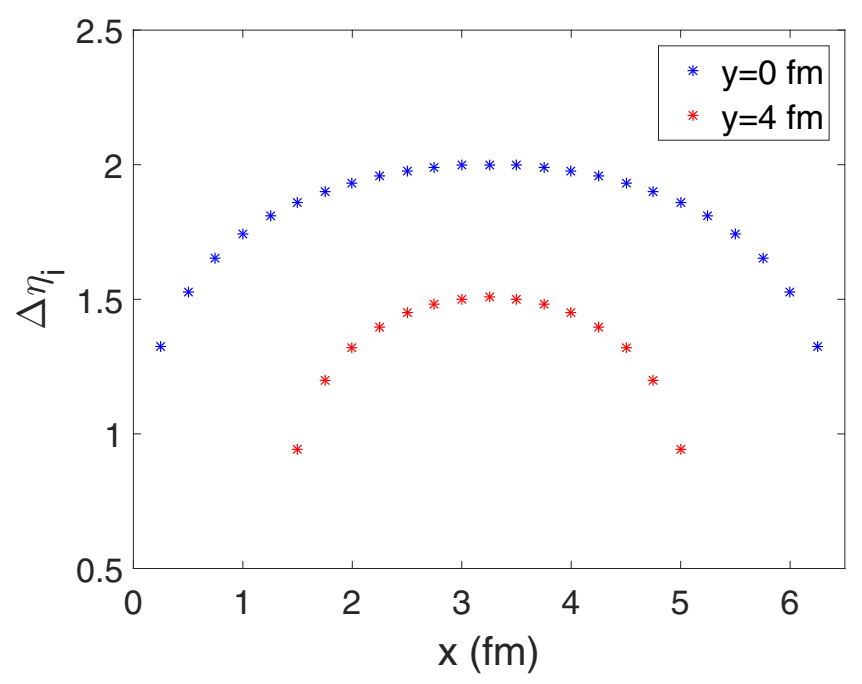

FIG. 7. The rapidity spread of the streaks $\Delta \eta_{i}$ in the reaction plane $[x, z]$ for $y=0$ (blue upper stars) and for $y=4 \mathrm{fm}$ (red down stars), for the same reaction and parameters listed in Fig. 4.

exactly satisfied by construction, at least at the moment $\tau_{i}=\tau_{0}$. Thus, our initial state model includes local shear and vorticity.

Our IS reflects qualitatively the behavior of the parton kinetic models: the blue (wider) streaks of Fig. 5 should be compared with Fig. 1.

The proper time evolution of the energy density and baryon density of the given streak is given by the following equations:

$$
\frac{d e_{i}}{d \tau_{i}}=-\frac{e_{i}+P_{i}}{\tau_{i}}, \quad \frac{d n_{i}}{d \tau_{i}}=-\frac{n_{i}}{\tau_{i}},
$$

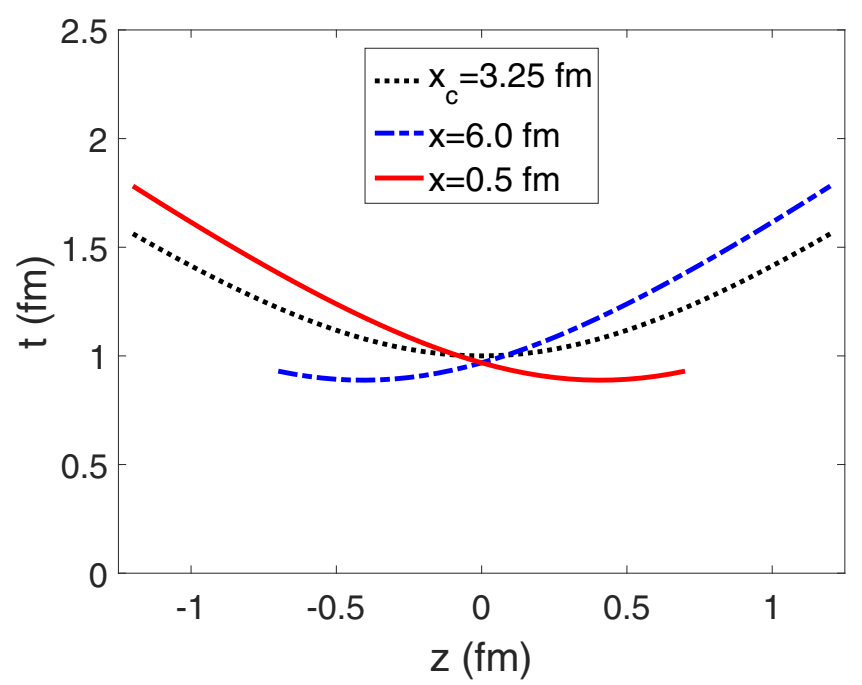

FIG. 8. Space-time $[t, z]$ configuration of the central streak, and two peripheral streaks, on the projectile and target sides. The origins of the streaks are not identical but at the proper time, $\tau_{0}$ the leading $(P)$ and trailing $(T)$ edge positions, $z_{\max }, z_{\min }$ match for the two side streaks, respectively. The two streaks cross each other in the spacetime at the points where both streaks have the same proper time $\tau_{0}$. The reaction parameters are the same as listed in Fig. 4. 


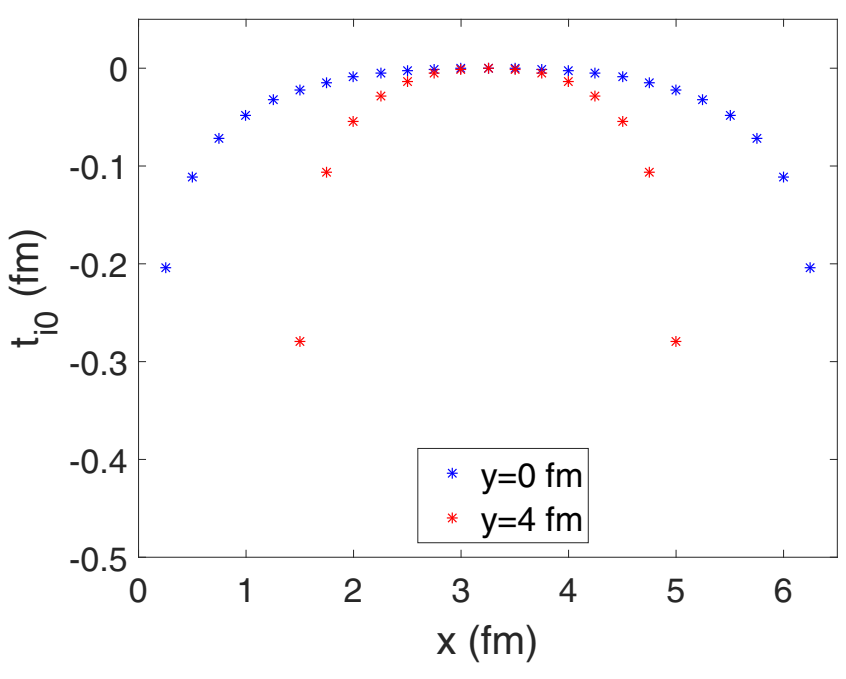

FIG. 9. The initial time coordinates, $t_{i 0}$, of the $i$ th streaks in the reaction plane $[x, z]$ for different values of $x$, for $y=0$ (blue upper stars) and for $y=4 \mathrm{fm}$ (red down stars). The reaction parameters are the same as listed in Fig. 4.

where the pressure $P_{i}$ is given by the equation of state, $P_{i}=$ $e_{i} / 3$. The initial conditions are given at $\tau_{i}=\tau_{0}, e_{i}\left(\tau_{0}\right), n_{i}\left(\tau_{0}\right)$ by Eqs. (26) and (33). This system can be solved easily:

$$
e_{i}\left(\tau_{i}\right)=e_{i}\left(\tau_{0}\right)\left(\frac{\tau_{0}}{\tau_{i}}\right)^{4 / 3}, \quad n_{i}\left(\tau_{i}\right)=n_{i}\left(\tau_{0}\right)\left(\frac{\tau_{0}}{\tau_{i}}\right)
$$

It is important to remember that if we want to have a finite volume fireball, we need to put some boundaries on the system. Here we assume that our system, i.e., given final streak $i$, described by the Bjorken model, is situated in the spa-

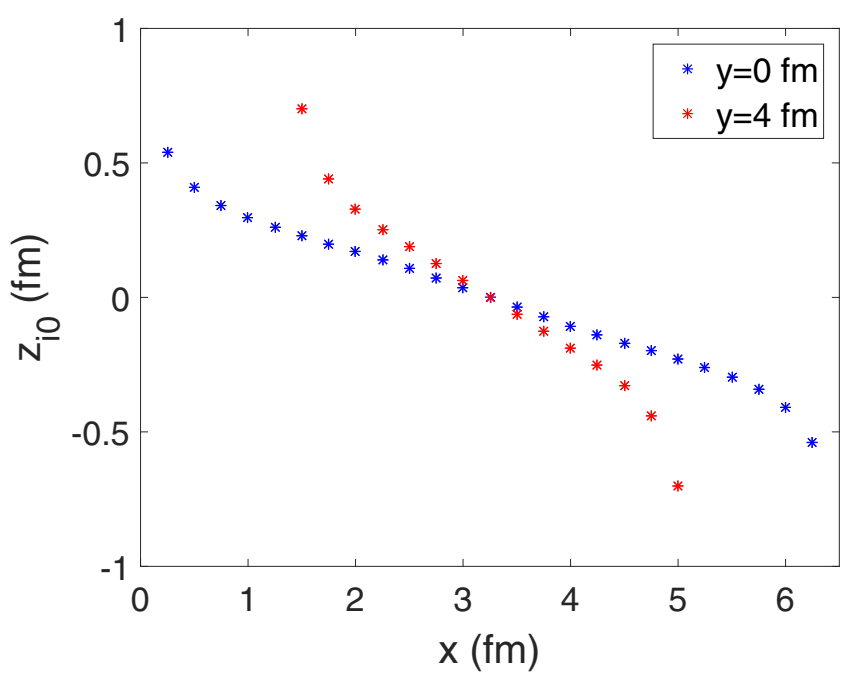

FIG. 10. The initial $z_{i 0}$ coordinates of the $i$ th streaks in the reaction plane $[x, z]$ for different values of $x$, for $y=0$ (blue stars distributed among wider $\mathrm{x}$ range $[0,6.5])$ and for $y=4 \mathrm{fm}$ (red stars distributed among narrower $x$ range $[1.5,5])$. The reaction parameters are the same as listed in Fig. 4. cial domain $\eta_{\min }^{i}<\eta_{i}<\eta_{\max }^{i}$. Within these boundaries the system is uniform along $\tau_{i}=$ const hyperbolae due to model assumptions, while outside we have vacuum with zero energy and baryon density as well as pressure. Thus, we have a jump, a discontinuity on the boundary, which remains during all the evolution. Certainly, to prevent matter expansion through such a boundary (due to strong pressure gradient) some work is done on the boundary surface [18]. One can think about it as putting some pressure to the surface with the vacuum, exactly the one which would remove discontinuity, then work is done by the expanding system against this pressure.

This actually means that although at the moment $\tau_{i}=\tau_{0}$ the energy density is taken in such a way that the energy and momentum conservation laws are satisfied, at any other moment of the proper time the energy is not explicitly conserved, because of the fixed $\eta$ boundaries: some is lost (for $\tau_{i}>\tau_{0}$ ), or it is also possible that some is gained (for $\tau_{i}<\tau_{0}$ ). Thus, for the overall collision IS, which we will be presenting in the following sections, at a given time in the laboratory system or at one global proper time, which will require some over different local $\tau_{i} \mathrm{~s}$, bigger or smaller than $\tau_{0}$, the total energy is strictly speaking not conserved. Although the difference is not that big for the IS parametrizations presented in the next section, since we are aware of this problem and trying to control it, the conservation laws are satisfied up to $3 \%$ of accuracy (usually better), which incudes also the errors coming from numerical gridding.

\section{IMPLEMENTATION IN CARTESIAN COORDINATES}

In our model at the first stage, each of the initial streaks lie on a different $\tau_{i}=\tau_{0}$ hyperbolae and is treated separately with the Bjorken model. The initial condition for each particular slabslab collision (at given transverse coordinates $x_{i}, y_{i}$ ) is given at $\tau_{i}=\tau_{0}$, but the proper times for each streak are different. To present the global initial state for the whole collision, the local baryon and energy densities along these streaks - as well as the local flow velocities-should be taken at some global $t=t_{\mathrm{IS}}=$ const. or $\tau=\tau_{\mathrm{IS}}=$ const. This means that for the given transverse coordinates one should calculate, for example, the energy density at different values of $\tau_{i}$, not only at one point. Therefore, the proper time propagation is done using the Bjorken hydrodynamical solution for the given slab-slab collision. In such a way we prepare an initial state for the overall collision, which resembles the parton cascade results. By so doing all conservation laws are preserved.

In principle the initial state can be defined on any timelike hypersurface, i.e., with any hypersurface with timelike normal vectors. In the general case this can be a complex curved timelike hypersurface; indeed, the PICR code, for example, allows the implementation of the curved IS hypersurface.

If the fluid dynamical code cannot handle a complex curved (in general case) timelike hypersurface and if the IS model and the FD model have different EoS, or if the IS model has no local equilibrium and therefore has no EoS, one has to use the matching conditions between the initial state reference frame and the fluid dynamical model's reference frame, as described in Ref. [19]. 
In this particular work we would like only to illustrate qualitatively the proposed new model for the IS, and therefore we will stay with the simplest choices of a timelike hypersurface for the transition from the initial state model to the fluid dynamics, which are $t=t_{\mathrm{IS}}=$ const. or $\tau=\tau_{\mathrm{IS}}=$ const.

The use of Milne coordinates $(\tau, x, y, \eta)$ in hydrodynamical calculations requires additional work that is outside the scope of this paper. The effects of an increasing cell size in the longitudinal direction during the calculation which leads to increasing numerical viscosity and dissipation as well as anisotropic viscosity are not understood. Analysis of these effects of a changing grid size on dissipation at relativistic energies is lacking and is much needed. Thus, using Cartesian coordinates with a constant and isotropic grid is advantageous for avoiding numerical anisotropy and other artifacts.

For a fluid dynamics (FD) model in Cartesian coordinates $(t, x, y, z)$ there is an obvious choice of using the laboratory or collider c.m. reference frame. We can define the transition surface between the IS model and the FD model in the Cartesian coordinates and can propagate the IS model solution up to this transition hypersurface. This will result in an initial state where the space-time points of the transition hypersurface do not have a constant $\tau_{i}$ from the origin of the $i$ th peripheral streak. Furthermore, each different peripheral streak, $i$, will have a different space-time origin.

Let us choose a constant time, $t=t_{\mathrm{IS}}=$ const., for the initial state hypersurface. Then we propagate (or cut) the initial state model up to this hypersurface from the initial $\tau_{i}=\tau_{0}$ hyperbolae. For a given $z_{\text {IS }}$ longitudinal coordinate of this $t=t_{\mathrm{IS}}$ hypersurface for the $i$ th streak the proper time from its origin, $\left[z_{i 0}, t_{i 0}\right]$ will be

$$
\tau_{i}^{\prime}\left(z_{\mathrm{IS}}\right)=\sqrt{\left(t_{\mathrm{IS}}-t_{i 0}\right)^{2}-\left(z_{\mathrm{IS}}-z_{i 0}\right)^{2}},
$$

where

$$
z_{\mathrm{IS}}=z_{i 0}+\left(t_{\mathrm{IS}}-t_{i 0}\right) \tanh \eta_{i},
$$

and $\eta_{i}$ can vary in the interval $\left[\eta_{\min }^{i, T}, \eta_{\max }^{i, P}\right]$.

Now using Bjorken hydrodynamic solution one can get the invariant scalar energy and baryon densities on this hyperbola:

$$
e_{\mathrm{IS}}\left(\tau_{i}^{\prime}\right)=e_{c}\left(\tau_{0}\right)\left(\frac{\tau_{0}}{\tau_{i}^{\prime}}\right)^{4 / 3} \text { and } n_{\mathrm{IS}}\left(\tau_{i}^{\prime}\right)=n_{i}\left(\tau_{0}\right)\left(\frac{\tau_{0}}{\tau_{i}^{\prime}}\right)
$$

We perform a simulation of the $\mathrm{Au}+\mathrm{Au}$ reaction at $100+$ $100 \mathrm{GeV} /$ nucl energy and impact parameter $b=0.5\left(R_{\mathrm{Pb}}+\right.$ $\left.R_{\mathrm{Pb}}\right)=6.5 \mathrm{fm}$, as shown in Fig. 4 . The model parameters are $\tau_{0}=1.0 \mathrm{fm}$ and $\Delta \eta_{c}=2$. We end our simulation of the IS for this reaction at a constant time hypersurface, $t_{\mathrm{IS}}=1.78 \mathrm{fm}$. This is actually a minimal possible time for such a calculation, namely $t_{\mathrm{IS}}=\operatorname{Max}\left\{t_{\max }^{i}\right\}$. Choosing any smaller $t_{\mathrm{IS}}$ would lead to the situation when some of $\tau_{i}^{\prime}$ s, calculated according to Eq. (42), would be smaller than the Bjorken initial state time $\tau_{0}$.

Our model with the above choice of parameters leads to a compact IS, substantially different from the IS of Refs. [1,2]: one can compare, for example the energy density distribution of Fig. 11 with Fig. 13(A) of Ref. [2]. As one can see in Figs. 11 and 12 the energy density in the middle region will be less due to the propagation to the corresponding larger proper time, $\tau_{i}^{\prime}$.
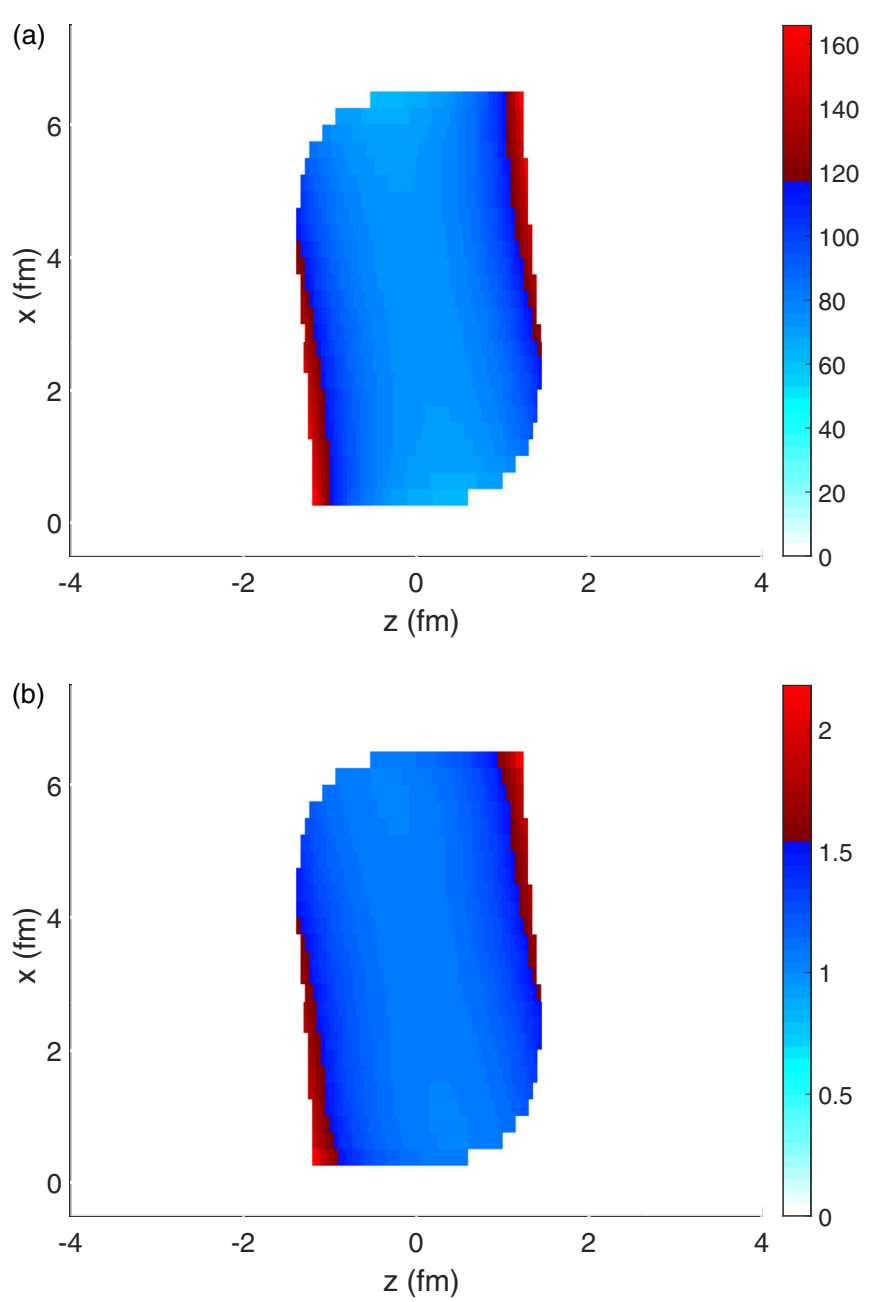

FIG. 11. The reaction plane, $y=0,[x, z]$ plot of the energy density (upper panel) and baryon density (lower panel) in units of $\mathrm{GeV} / \mathrm{fm}^{3}$ and $1 / \mathrm{fm}^{3}$ correspondingly, propagated to the constant time, $t=t_{\mathrm{IS}}=1.78 \mathrm{fm} / \mathrm{c}$ hypersurface. The resulting density distributions shows a maxima at the both the forward and backward edges of the reaction plane. Although the energy density and baryon density are uniform at $\tau_{i}=\tau_{0}$ for each streak in its own frame as shown in Fig. 4, the observed space-time dependence arises from the propagation to the $t=t_{\mathrm{IS}}$ hypersurface. The reaction parameters are the same as listed in Fig. 4.

The structure of the net baryon density distribution is very similar to the energy density distribution, as shown in Fig. 11. The propagated net baryon density shows maxima at the forward and backward edges of the matter in the reaction plane. The maximum value of the nucleon number is $n \approx 2.7 \mathrm{fm}^{-3}$.

As one can see in Fig. 13 the present model shows considerable shear in the velocity field. In the center of the reaction plane, in the direction of the impact parameter vector, $x$, the upper (positive) side shows a forward motion (positive velocities $v$ ) while the lower side shows negative velocities. Further forward in the beam direction (at $z=0.5 \mathrm{fm}$ ), the velocity profile is identical but shifted in velocity to higher positive values due to the longitudinal Bjorken expansion on the model. On the opposite side (at $z=-0.5 \mathrm{fm}$ ) the shift is opposite due to the Bjorken expansion. 


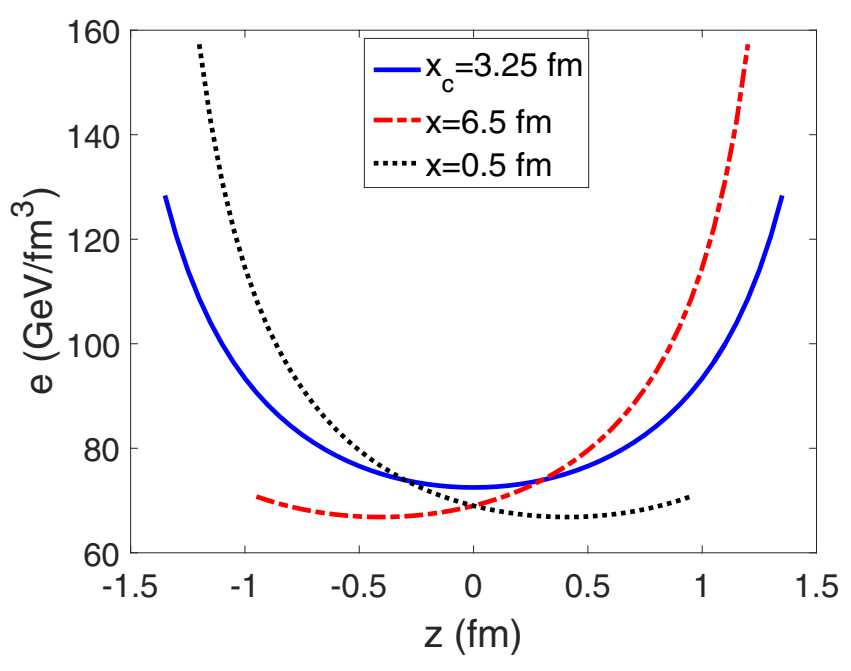

FIG. 12. The energy density distribution along the $z$ direction, for the central streak at $x=3.25 \mathrm{fm}$ and for the peripheral streaks at $x=0.5$ and $6.5 \mathrm{fm}$. The matter of fluid elements was propagated to the constant time, $t_{\mathrm{IS}}=1.78 \mathrm{fm} / \mathrm{c}$, hypersurface. This example is calculated for the same reaction and parameters that are listed in Fig. 4.

The side layers that are parallel to the reaction plane at finite $y$ values, show the same shear flow profile, but with higher shear, $\partial v_{z} / \partial x$, see Fig. 14.

\section{IMPLEMENTATION IN MILNE COORDINATES $\tau, X, Y, \eta$}

We now show how our IS can be implemented in a fluid dynamical model in $x, y, \eta, \tau$ coordinates. In these coordinates the numerical solution can be performed in the frame of the central streak. In other words, our model will give an

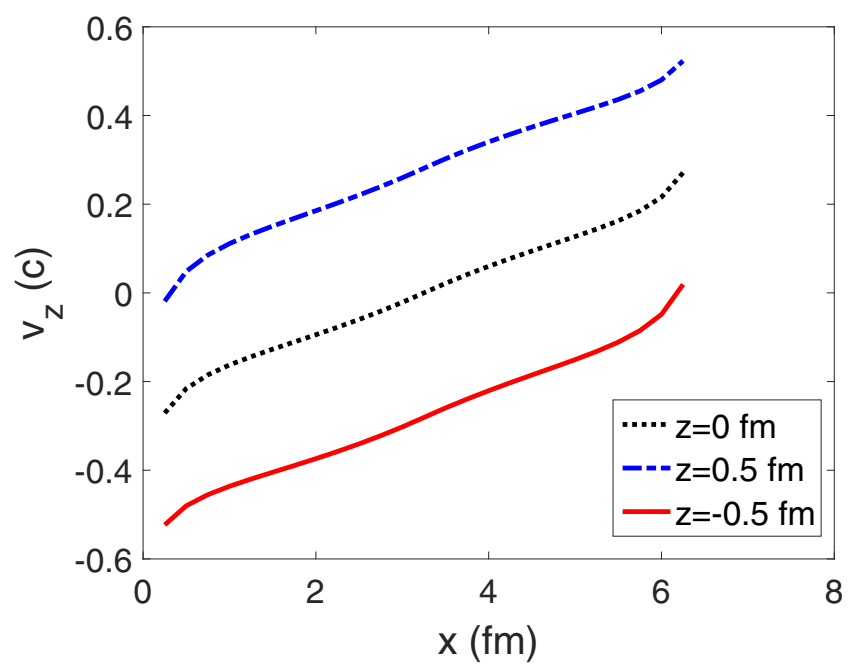

FIG. 13. The $z$-directed velocity distribution versus the $x$ position (at $y=0$ ), in the $z=0 \mathrm{fm}$ central plane (dash line) propagated to the constant time, $t_{\mathrm{IS}}=1.78 \mathrm{fm} / \mathrm{c}$ hypersurface. The velocity distribution for the $z= \pm 0.5 \mathrm{fm}$ forward/backward shifted positions are shown by dash-dotted and solid lines, respectively. The reaction parameters are the same as listed in Fig. 4.

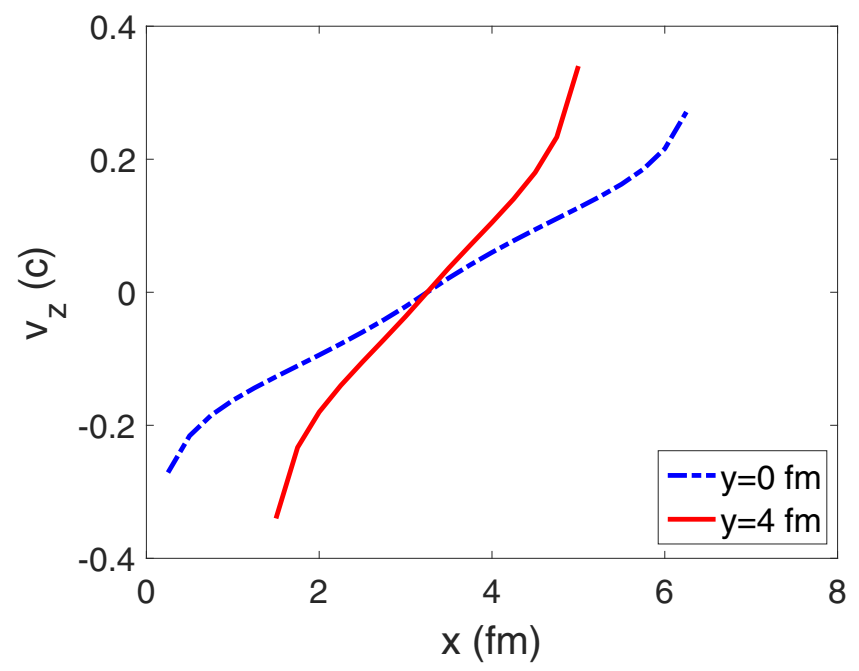

FIG. 14. The velocity, $v_{z}$, distribution in $x$ direction (at $z=0$ ), for the central layer, $y=0 \mathrm{fm}$ (dash-dot line), and for the peripheral layer at $y=4 \mathrm{fm}$ (solid line), propagated to the constant time, $t_{\mathrm{IS}}=$ $1.78 \mathrm{fm} / \mathrm{c}$ hypersurface. The reaction parameters are the same as listed in Fig. 4. Interestingly the longitudinal shear among the neighboring peripheral $x$ layers is much bigger than in the center.

initial state for further FD evolution on the $\tau_{c}=\tau_{\mathrm{IS}}=$ const. hypersurface.

Using the c.m. $x, y, \eta_{c}, \tau_{c}$ coordinates one can calculate for each space-time point of the hypersurface the corresponding $x, y, z, t$ coordinates using Eqs. (1), and then relating those with any $x, y, \eta_{i}, \tau_{i} \mathrm{RFS}_{i}$ frame.

In the central streak frame for any point on a $\tau_{c}=\tau_{\text {IS }}$ hyperbola we have $\left(t_{c 0}=0, z_{c 0}=0\right)$

$$
t_{\mathrm{IS}}=\tau_{\mathrm{IS}} \cosh \eta_{c}, \quad z_{\mathrm{IS}}=\tau_{\mathrm{IS}} \sinh \eta_{c} .
$$

In the frame of the $i$ th side streak, however,

$$
\begin{aligned}
\tau_{i}^{\prime} & =\sqrt{\left(t_{\mathrm{IS}}-t_{i 0}\right)^{2}-\left(z_{\mathrm{IS}}-z_{i 0}\right)^{2}} \\
& =\sqrt{\left(\tau_{\mathrm{IS}} \cosh \eta_{c}-t_{i 0}\right)^{2}-\left(\tau_{\mathrm{IS}} \sinh \eta_{c}-z_{i 0}\right)^{2}},
\end{aligned}
$$

and the corresponding rapidity in the $i$ th peripheral frame is

$$
\eta_{i}^{\prime}=\operatorname{Artanh}\left(\frac{\tau_{\text {IS }} \sinh \eta_{c}-z_{i 0}}{\tau_{\text {IS }} \cosh \eta_{c}-t_{i 0}}\right) .
$$

We know that for each streak $i$, the geometrical rapidity should be within the limits $\left[\eta_{\min }^{i}, \eta_{\max }^{i}\right]$. Imposing these conditions on the $\eta_{i}^{\prime}$, given by Eq. (47), one can find the corresponding limits for the $\eta_{c}$ for the $i$ th streak:

$$
\eta_{c} \leqslant \eta_{\max }^{i}+\operatorname{Arsinh}\left(\frac{z_{i 0} \cosh \eta_{\max }^{i}-t_{i 0} \sinh \eta_{\max }^{i}}{\tau_{\mathrm{IS}}}\right)
$$

and

$$
\eta_{c} \geqslant \eta_{\min }^{i}+\operatorname{Arsinh}\left(\frac{z_{i 0} \cosh \eta_{\text {min }}^{i}-t_{i 0} \sinh \eta_{\min }^{i}}{\tau_{\mathrm{IS}}}\right) .
$$

The detailed discussion on propagation and rapidity limits can be seen in the Appendix. 


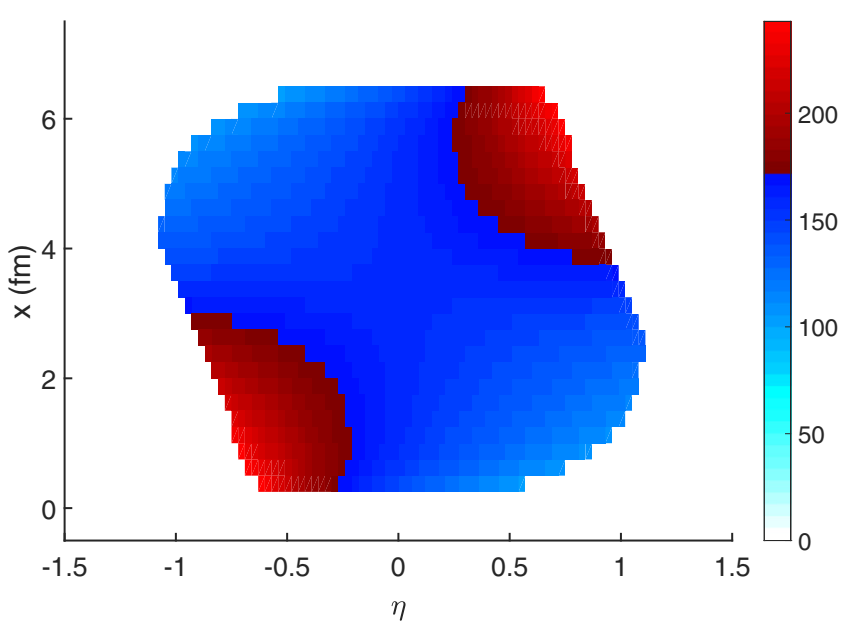

FIG. 15. The reaction plane $y=0,[x, z]$ plot of the energy density, $e(x, \eta)$, in units of $\mathrm{GeV} / \mathrm{fm}^{3}$, propagated to the constant proper time, $\tau_{c}=\tau_{0}=1.0 \mathrm{fm} / \mathrm{c}$ hypersurface. The $\left(\tau_{i}, \eta_{i}\right)$ points of each particular streak are propagated forward and backward to the overall $\tau_{c}=1.0 \mathrm{fm} / \mathrm{c}$ hyperbola, as explained in the text. The propagated initial density shows maximal energy densities of $e \approx 240 \mathrm{GeV} / \mathrm{fm}^{3}$ at the forward and backward edges of the reaction plane, although $e_{c}\left(\tau_{0}\right)=156.31 \mathrm{GeV} / \mathrm{fm}^{3}$. The reaction parameters are the same as listed in Fig. 4.

Now with $\tau_{i}^{\prime}$, given by Eq. (46), we can calculate in the IS model energy and baryon densities at the pretransition side of the IS/FD transition hypersurface; see Eqs. (44).

The quantity $\tau_{\mathrm{IS}}$ is a parameter of our model. For illustrative purposes of this particular study we have chosen $\tau_{\mathrm{IS}}=\tau_{0}$.

The resulting energy density distribution is shown in Figs. 15 and 16. For the central streak it is now flat and equal to $e_{c}\left(\tau_{0}\right)=156.31 \mathrm{GeV} / \mathrm{fm}^{3}$. The noncentral streaks show a strong asymmetry peaking forward or backward. The propagated initial density shows maximal energy densities of $e \approx 240 \mathrm{GeV} / \mathrm{fm}^{3}$ at the forward and backward edges of the reaction plane. Such high densities, higher than the energy density at $\tau_{i}=\tau_{0}\left[e_{i}\left(\tau_{0}\right)=156.31 \mathrm{GeV} / \mathrm{fm}^{3}\right]$, are reached, because for the $\tau_{\mathrm{IS}}=\tau_{0}$ IS hypersurface the $\tau_{i}^{\prime} \mathrm{s}$, calculated according to Eq. (46), can be smaller than the Bjorken initial state time $\tau_{0}$, and thus $e_{\mathrm{IS}}\left(\tau_{i}^{\prime}\right)=e_{c}\left(\tau_{0}\right)\left(\frac{\tau_{0}}{\tau_{i}^{\prime}}\right)^{4 / 3}>e_{c}\left(\tau_{0}\right)$. For the above formulas it is assumed that the Bjorken solution is formally valid even in the pre-equilibrium stages of the reaction $\tau_{i}^{\prime}<\tau_{0}$. Such an assumption may be questionable, but in this work we only aim to illustrate our initial state model and follow it for simplicity.

The overall energy density distribution is a bit smoother for the $\tau=$ const. IS than for the $t=$ const. IS. This can be clearly seen comparing Figs. 12 and 16.

The flow velocity distribution in the final streaks along the beam axis as a function of $\eta$ is shown in Fig. 17 .

\section{CONCLUSIONS AND DISCUSSION}

In the present work we propose a new initial state model for hydrodynamic simulation of relativistic heavy ion collisions based on Bjorken-like solutions applied streak by streak in the

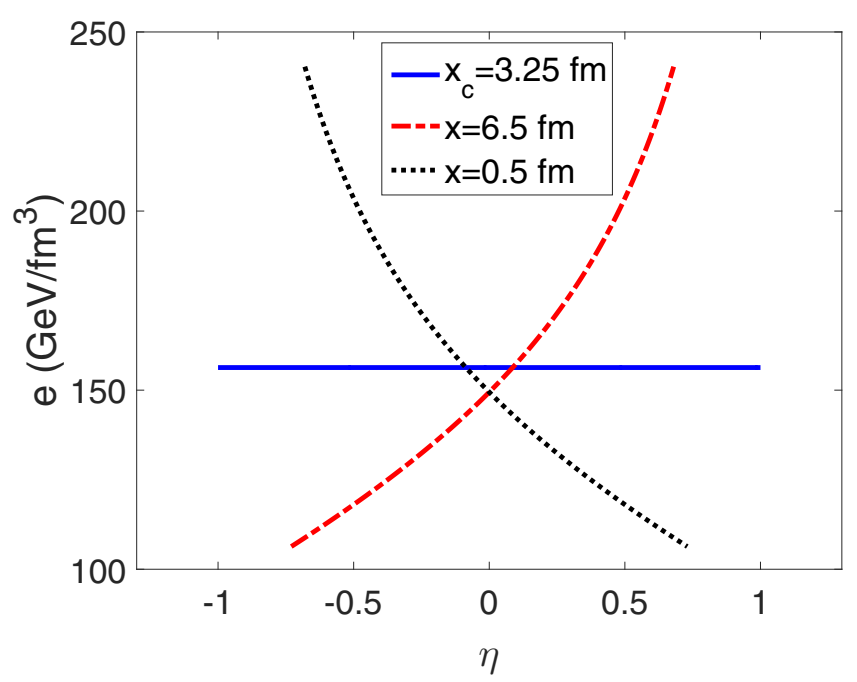

FIG. 16. The energy density, $e$, distribution versus $\eta$, for the central streak at $x_{c}=3.25 \mathrm{fm}$ and for peripheral streaks at $x=6.5$ and $0.5 \mathrm{fm}$, propagated to the constant proper time, $\tau_{c}=1.0 \mathrm{fm} / \mathrm{c}$ hypersurface. The propagated initial density shows maximal and minimal energy densities at the forward and backward edges of the peripheral streaks. The reaction parameters are the same as listed in Fig. 4.

transverse plane and producing an IS qualitatively similar to the results of parton cascade models like Ref. [7]. Our IS can be given in both $[t, x, y, z]$ and $[\tau, x, y, \eta]$ coordinates, and thus can be tested by all $3+1$ D hydrodynamical codes which exist in the field. Most importantly, it is able to incorporate initial shear, in contrary to several other initial state parametrizations. The lack of initial shear reduces the vorticity and the possibility

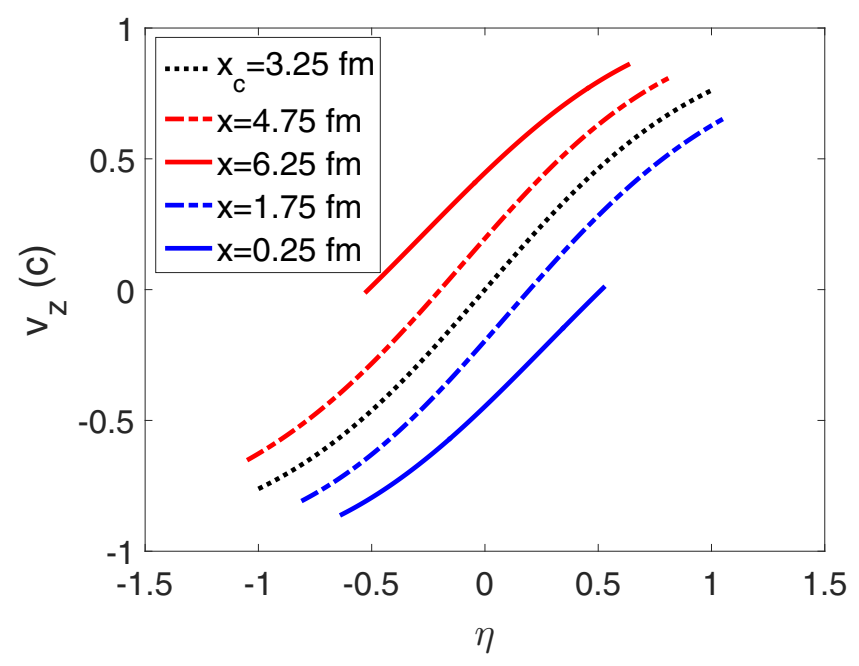

FIG. 17. The $z$-directed velocity distribution versus the $\eta$ coordinate (at $y=0$ ), for the central streak (at $x_{c}=3.25 \mathrm{fm}$ ). Semiperipheral streaks $(x=1.75$ and $4.75 \mathrm{fm})$ and peripheral streaks $(x=$ 0.25 and $6.25 \mathrm{fm}$ ), propagated to the constant proper time, $\tau_{c}=1.0$ $\mathrm{fm} / \mathrm{c}$ hypersurface are also shown. The reaction parameters are the same as listed in Fig. 4. 


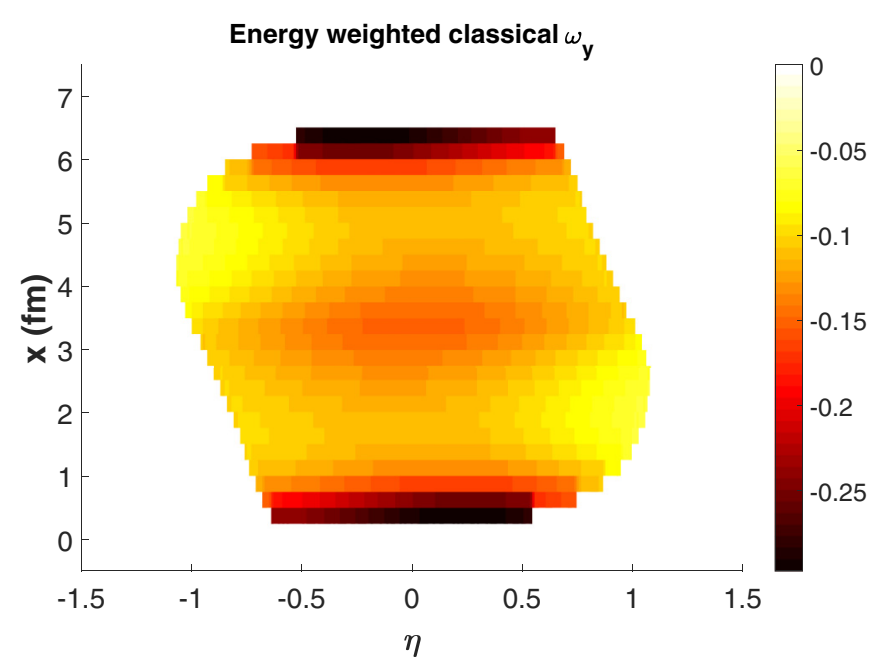

FIG. 18. The $y$ component of the classical energy weighted vorticity on the $[x, \eta]$ plane, at $y=0$. This is the dominant component of vorticity, and it points everywhere in the $-y$ direction. This example is obtained by propagation of the initial configuration to the hypersurface at $\tau_{c}=\tau_{0}=1.0 \mathrm{fm} / \mathrm{c}$. The at the upper and lower edges the vorticity approaches -0.3 .

for polarization in those models, which contradicts recent observations $[5,6]$.

The velocity distributions produced in our initial state model are shown in Figs. 13, 14, and 17 in the reaction plane, $y=0$. As we see among the different streaks of the matter there is considerable shear, particularly for peripheral streaks, e.g., $y=4 \mathrm{fm}$; see Fig. 14. Figure 17 indicates that the velocity profile shows dominant longitudinal expansion, which gradually may decrease the central shear. Thus, the development of a Kelvin-Helmholtz instability in this configuration is less probable than in earlier calculations with a different initial state [20].

The shear will lead to strong vorticity. This vorticity vector pointing in the $-y$ direction, dominates the vorticities developing due to the expansion later in the flow. Furthermore, due to symmetry reasons the vorticities in the other directions cancel each other to a large extent $[21,22]$, except for eventual unbalanced vorticities due to random fluctuations [23,24].

The energy weighted classical vorticity, $\omega_{y}(x, \eta)$, see Ref. [4] for detailed definition, is shown in Fig. 18. This component is overall negative arising from the initial rotation, i.e., it is pointing in the $-y$ direction. The central part of the momentum domain at this initial moment shows smaller vorticity, due to the Bjorken-like expansion of the model.

The classical vorticity, $\omega_{x}(y, \eta)$ is shown in Fig. 19. This component is antisymmetric across the $y=0$ surface. As a consequence the contribution of this component vanishes in the complete averaging. The central part of the domain at this initial moment shows smaller vorticity.

The vorticity is observed via the observed polarization, $\Pi_{y}$, of emitted $\Lambda$ and $\bar{\Lambda}$ particles $[13,15,25]$. The symmetries of the vorticity field and of the momentum dependence of the polarizations are tightly related [25]. The present experiments show only the overall $\Lambda$ and $\bar{\Lambda}$ polarizations summed up for

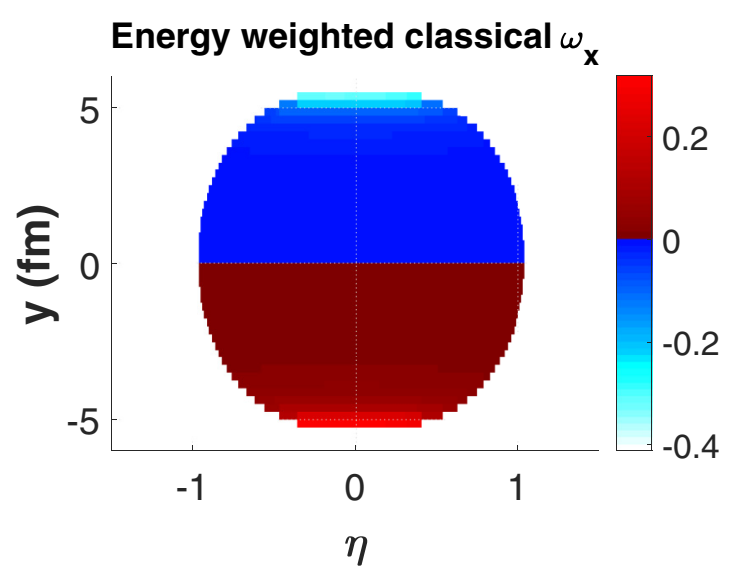

FIG. 19. The $x$ component of the classical vorticity on the $[y, \eta]$ plane at $x=3.0 \mathrm{fm}$. This component of vorticity has similar values as the $y$ component, but it is antisymmetric, $\omega_{x}\left(y_{\max }, \eta=0\right)=$ $-\omega_{x}\left(y_{\min }, \eta=0\right)$. So the two identical but opposite signed vorticities yield a vanishing overall sum.

all emission momenta. Thus, in the c.m. frame the polarization components $\Pi_{x}$ and $\Pi_{z}$ must vanish, due to the symmetries of vorticity components, $\omega_{x}$ and $\omega_{z}$, except a smaller contribution from random fluctuations.

At the same time the $x$ and $y$ vorticity components carry valuable information, but these can only be extracted if the participant c.m. is identified event by event (EbE) [26,27]. This identification based on the spectators detected via the zero degree calorimeters is not performed yet experimentally [28], due to assumed, unrelated fluctuations of other origins. Now this identification method could be tested by evaluating the sum of polarizations, $\Pi_{x}$ and $\Pi_{z}$, with and without $\mathrm{EbE}$ identification of participant c.m. With c.m. identification the $x$ and $y$ polarization components should vanish or become minimal. The $\Pi_{x}(\mathrm{p})$ and $\Pi_{z}(\mathrm{p})$ distributions should also show the symmetries arising from the symmetries of the vorticities. This will provide valuable information on the details of the initial state models which cannot be easily detected in other ways.

The current model is a simple realization for peripheral heavy-ion collisions, with initial shear and vorticity, in Milne coordinates. Unlike the large majority of the Bjorken type of models that do not discuss the longitudinal degrees of freedom, we divide the transverse plane to streaks that are longitudinally finite. At every transverse point $i \equiv\left[x_{i}, y_{i}\right]$ we have a longitudinal streak with well defined end points, $z_{i, P}^{\max }$ and $z_{i, P}^{\min }$ or the corresponding points in Milne coordinates $\eta_{i, P}^{\max }$ and $\eta_{i, P}^{\min }$ on the projectile side. We describe the target side similarly. We obtain these points from the streak by streak energy and momentum conservation and from simple assumptions regarding the streak ends and streak center points.

There exist a few models in Milne coordinates, which discuss the longitudinal degrees of freedom in the collisions, and satisfy energy and momentum conservations. For example, Ref. [17] introduces streak ends, $z_{p}(\tau)$ and $z_{t}(\tau)$, but these are uniform, i.e., identical for all transverse points. This model could be generalized in the same way to varying peripheral 
streaks, so that energy and momentum conservation is applied streak by streak, and as a consequence shear and vorticity will be included in the model. In this case every transverse streak would have a different constant proper time hyperbola, with different origins in the space-time $\left[t_{i 0}, z_{i 0}\right]$.

A very interesting approach has been presented at the Quark Matter 2017 Conference [29]. The whole model is still in preparation, but according to the figures in Ref. [29] this model also has asymmetric hyperbolae, which appear as "thermalized strings." As far as we understood the asymmetry in this model is related to IS fluctuations in the position/time of the initial parton collisions, and it is not clear whether it is systematically increasing with $x$, as in our case. Also we would like to note that the model of Ref. [29] has zero pressure free streaming before thermalization, which may lead to unrealistically increasing transparency and may eliminate the development of local vorticity in peripheral collisions. This is in stark contrast to the field dominant initial state dynamics described in Refs. [1,2,16,30,31].

In case of color glass condensate in the initial state, the color field slows down the leading charges of the expanding system, as discussed in Refs. [32-35]. One can follow the trajectory of the longitudinal edges up to some $\tau=$ const. hypersurface and obtain the corresponding space-time rapidities $\eta_{\min }$ and $\eta_{\max }$, which limit the longitudinal extent of the flux-tube with the gluon field or plasma. The field may even contribute to a large compression of the baryon charge at the forward and backward edges $[30,36]$. This model could also be generalized to varying peripheral streaks, so that energy and momentum conservation is applied streak by streak and the streak ends would be different for each peripheral streak.

And finally we would like to mention that by varying the parameters of our model, namely $\Delta \eta_{c}, \tau_{0}, t_{\mathrm{IS}}$ or $\tau_{\mathrm{IS}}$, the geometry of the produced IS can be adjusted to the different parton cascade approaches as well as to the different field theoretical models. For example, using parameter set $\Delta \eta_{c}=$ $1.7, \tau_{0}=2.0 \mathrm{fm} / \mathrm{c}, t_{\mathrm{IS}}=3.2 \mathrm{fm} / \mathrm{c}$ we managed to reproduce rather closely the form and volume of the IS from Ref. [2] (of course, the flow velocity and energy density distributions are still fixed by the Bjorken nature of the model and stay rather different from those in Ref. [2]). This feature of the proposed approach may provide a basis for further studies of different physical processes.

\section{ACKNOWLEDGMENTS}

Enlightening discussions with Hannah Petersen and Etele Molnar are gratefully acknowledged. Y. L. Xie is supported by China Scholarship Council (China) and by COST Action Grant No. COST-STSM-ECOST-STSM-CA15213-080317-084442. This work was partially supported by by the Alexander von Humboldt Foundation, by the Research Council of Norway, Grant No. 255253/F50, by the Spanish Ministerio de Economia y Competitividad (MINECO) under Project No. MDM-20140369 of ICCUB (Unidad de Excelencia "María de Maeztu”), Generalitat de Catalunya, Contract No. 2014SGR-401, and, with additional European FEDER funds, under Contract No. FIS2014-54762-P and the Spanish Excellence Network on Hadronic Physics Grant No. FIS2014-57026-REDT.

\section{APPENDIX: PROPAGATION TO THE CENTRAL STREAK FRAME}

As we have discussed in Sec. V, after Bjorken expansion, the central streak and $i$ th peripheral streak, with different initial points, will stop at the $\tau_{c}=\tau_{i}=\tau_{\mathrm{IS}}$ hyperbolae, which are shown in Fig. 20. Now we map the solution of $\tau_{i}=\tau_{\mathrm{IS}}$ to the $\tau_{c}=\tau_{\text {IS }}$ hyperbola, which we call "propagation," by keeping the rapidity $\eta_{i}$ unchanged. That is, from Eq. (47) the point $\left(t_{i}, z_{i}\right)$ in Fig. 20 , with its propagated point $\left(t_{\mathrm{IS}}, z_{\mathrm{IS}}\right)$ will have the same rapidity in the $i$ th peripheral streak's frame:

$$
\eta_{i}=\eta_{i}^{\prime}=\operatorname{Artanh}\left(\frac{\tau_{\mathrm{IS}} \sinh \eta_{c}-z_{i 0}}{\tau_{\mathrm{IS}} \cosh \eta_{c}-t_{i 0}}\right) .
$$

This equation combined with Eqs. (45) and (46) describe the propagation, see Fig. 20.

Now from assumption (a), we have limits on $z$ coordinate of the $i$ th streak: $z_{\max }^{i, P}=z_{\max }^{c}$ and $z_{\text {min }}^{i, T}=z_{\min }^{c}$, which results on limits on rapidity, i.e., $\eta_{\text {min }}^{i} \leqslant \eta_{i} \leqslant \eta_{\max }^{i}$. Imposing these conditions on the $\eta_{i}^{\prime}$, given by Eq. (A1), we can find the corresponding limits for the $\eta_{c}$ for the $i$ th streak.

Thus,

$$
\tanh \eta_{i}^{\prime}=\left(\frac{\tau_{\mathrm{IS}} \sinh \eta_{c}-z_{i 0}}{\tau_{\mathrm{IS}} \cosh \eta_{c}-t_{i 0}}\right) \leqslant \tanh \eta_{\max }^{i},
$$

where $\eta_{\max }^{i}=\left\langle\eta_{i}\right\rangle+\frac{\Delta \eta_{i}}{2}$, calculated as explained in Sec. III. Multiplying the second inequality by [cosh $\left.\eta_{\max }^{i} \cdot\left(\tau \cosh \eta_{c}-t_{i 0}\right)\right]$, we get

$\left(\tau_{\mathrm{IS}} \sinh \eta_{c}-z_{i 0}\right) \cosh \eta_{\max }^{i} \leqslant\left(\tau_{\mathrm{IS}} \cosh \eta_{c}-t_{i 0}\right) \sinh \eta_{\max }^{i}$.

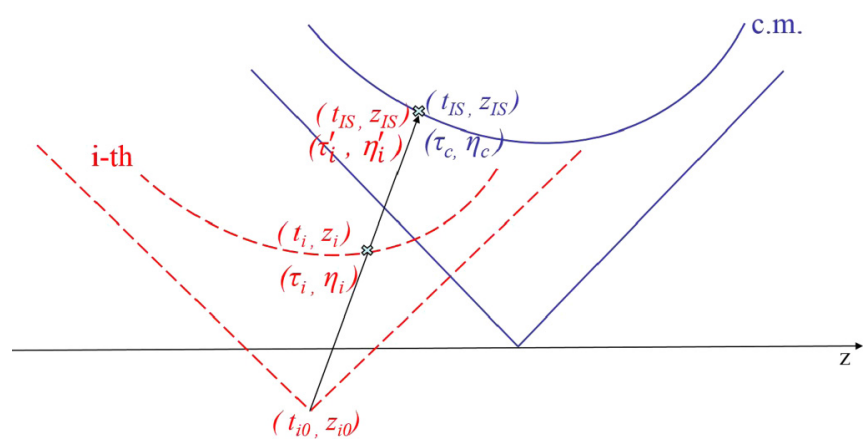

FIG. 20. The propagation from the $i$ th peripheral hyperbola to the central streak hyperbola, in order that all streaks will be situated on a joint (blue solid) hyperbola at $\tau_{c}=\tau_{\mathrm{IS}}=$ const. The primary situation of the initial state is on the (red dashed) $i$ th peripheral hyperbola at $\left(t_{i}, z_{i}\right)$ or $\left(\tau_{i}, \eta_{i}\right)$. With the same rapidity, $\eta_{i}$, this fluid element is propagated to the (blue solid) c.m. hyperbola to point $\left(t_{\mathrm{IS}}, z_{\mathrm{IS}}\right)$ or $\left(\tau_{i}^{\prime}, \eta_{i}^{\prime}\right)$. The same point in the c.m. frame is on the (blue solid) central streak hyperbola at the same space-time coordinates $\left(t_{\mathrm{IS}}, z_{\mathrm{IS}}\right)$, but in the (blue solid) frame's coordinates it is $\left(\tau_{c}, \eta_{c}\right)$. 
Performing the multiplications, and using the expression of $\sinh (A-B)$, we obtain

$$
\sinh \left(\eta_{c}-\eta_{\max }^{i}\right) \leqslant \frac{z_{i 0} \cosh \eta_{\max }^{i}-t_{i 0} \sinh \eta_{\max }^{i}}{\tau_{\mathrm{IS}}}
$$

Now taking Arsinh of this equation leads to Eq. (48):

$$
\eta_{c} \leqslant \eta_{\max }^{i}+\operatorname{Arsinh}\left(\frac{z_{i 0} \cosh \eta_{\text {max }}^{i}-t_{i 0} \sinh \eta_{\max }^{i}}{\tau_{\mathrm{IS}}}\right) .
$$

Similarly, one can get Eq. (49).
[1] V. K. Magas, L. P. Csernai, and D. D. Strottman, Phys. Rev. C 64, 014901 (2001).

[2] V. K. Magas, L. P. Csernai, and D. D. Strottman, Nucl. Phys. A 712, 167 (2002).

[3] L. P. Csernai, V. K. Magas, H. Stocker, and D. D. Strottman, Phys. Rev. C 84, 024914 (2011).

[4] L. P. Csernai, V. K. Magas, and D. J. Wang, Phys. Rev. C 87, 034906 (2013).

[5] M. A. Lisa et al. (STAR Collaboration), Invited talk presented at the QCD Chirality Workshop 2016, Feb. 23-26, 2016, Los Angeles, USA.

[6] L. Adamczyk et al. (The STAR Collaboration), Nature 548, 62 (2017).

[7] L. G. Pang, H. Petersen, G. Y. Qin, V. Roy, and X. N. Wang, Nucl. Phys. A 956, 272 (2016).

[8] L.-G. Pang, H. Petersen, Q. Wang, and X.-N. Wang, Phys. Rev. Lett. 117, 192301 (2016); L. G. Pang, R. H. Fang, H. Petersen, Q. Wang, and X. N. Wang, J. Phys. Conf. Ser. 779, 012069 (2017); H. Li, H. Petersen, L. G. Pang, Q. Wang, X. L. Xia, and X. N. Wang, Nucl. Phys. A 967, 772 (2017).

[9] D.-m. Zhou, Y.-1. Yan, X.-1. Li, X.-m. Li, B.-g. Dong, X. Cai, and B.-h. Sa, Comput. Phys. Commun. 193, 89 (2015).

[10] B. Zhang, C. M. Ko, B.-A. Li, and Z. Lin, Phys. Rev. C 61, 067901 (2000).

[11] W. T. Deng and X. G. Huang, J. Phys. Conf. Ser. 779, 012070 (2017).

[12] V. Vovchenko, I. A. Karpenko, M. I. Gorenstein, L. M. Satarov, I. N. Mishustin, B. Kampfer, and H. Stoecker, Phys. Rev. C 94, 024906 (2016); V. Vovchenko, L.-G. Pang, H. Niemi, Iu. A. Karpenko, M. I. Gorenstein, L. M. Satarov, I. N. Mishustin, B. Kämpfer, H. Stocker, PoS BORMIO 2016, 039 (2016); V. Vovchenko, M. I. Gorenstein, L. M. Satarov, I. N. Mishustin, L. P. Csernai, I. Kisel, and H. Stöcker, Phys. Rev. C 93, 014906 (2016).

[13] F. Becattini, L. P. Csernai, and D. J. Wang, Phys. Rev. C 88, 034905(R) (2013).

[14] L. P. Csernai, D. J. Wang, M. Bleicher, and H. Stöcker, Phys. Rev. C 90, 021904(R) (2014).

[15] F. Becattini, L. P. Csernai, D. J. Wang, and Y. L. Xie, Phys. Rev. C 93, 069901(E) (2016).
[16] I. N. Mishustin and J. I. Kapusta, Phys. Rev. Lett. 88, 112501 (2002); I. N. Mishustin and K. A. Lyakhov, Phys. Rev. C 76, 011603 (2007).

[17] I. N. Mishustin and K. A. Lyakhov, Phys. At. Nucl. 75, 371 (2011).

[18] K. J. Eskola, K. Kajantie, and P. V. Ruuskanen, Eur. Phys. J. C 1, 627 (1998); V. K. Magas and L. P. Csernai, Phys. Lett. B 663, 191 (2008).

[19] Y. Cheng, L. P. Csernai, V. K. Magas, B. R. Schlei, and D. Strottman, Phys. Rev. C 81, 064910 (2010).

[20] L. P. Csernai, D. D. Strottman, and C. Anderlik, Phys. Rev. C 85, 054901 (2012).

[21] Y. L. Xie, M. Bleicher, H. Stöcker, D. J. Wang, and L. P. Csernai, Phys. Rev. C 94, 054907 (2016).

[22] Y. L. Xie, D. J. Wang, and L. P. Csernai, Phys. Rev. C 95, 031901(R) (2017).

[23] S. Floerchinger and U. A. Wiedemann, Phys. Rev. C 88, 044906 (2013).

[24] S. Floerchinger and U. A. Wiedemann, J. High Energy Phys. (2011) 100; J. Phys. G: Nucl. Part. Phys. 38, 124171 (2011).

[25] F. Becattini, V. Chandra, L. Del Zanna, and E. Grossi, Ann. Phys. 338, 32 (2013).

[26] L. P. Csernai, G. Eyyubova, and V. K. Magas, Phys. Rev. C 86, 024912 (2012).

[27] L. P. Csernai and H. Stöcker, J. Phys. G 41, 124001 (2014).

[28] J. Schukraft (private communication).

[29] C. Shen, G. Denicol, C. Gale, S. Jeon, A. Monnai, and B. Schenke, Nucl. Phys. A 967, 796 (2017).

[30] M. Gyulassy and L. P. Csernai, Nucl. Phys. A 460, 723 (1986).

[31] H. Stoecker et al., J. Phys. G 43, 015105 (2016).

[32] R. J. Fries, J. I. Kapusta, and Y. Li, Nucl. Phys. A 774, 861 (2006).

[33] S. Ozonder and R. J. Fries, Phys. Rev. C 89, 034902 (2014).

[34] G. Chen, R. J. Fries, J. I. Kapusta, and Y. Li, Phys. Rev. C 92 , 064912 (2015).

[35] R. J. Fries, G. Chen, and S. Somanathan, Phys. Rev. C 97, 034903 (2018).

[36] M. Li and J. I. Kapusta, Phys. Rev. C 95, 011901(R) (2017).

Correction: The email address was missing for the corresponding author at publication; the footnote has now been inserted. 